\title{
Cytogenetic and biochemical studies on the effect of DDB in albino mice and their embryos.
}

\author{
Adel El Rokeeb and Amira Abd El Raouf \\ AL. Azhar University Faculty of Medicine \\ (Boys branch) Egypty \\ National Research Centre Cell Biology Department Dokki - Egypt
}

\begin{abstract}
Introduction: DDB (Dimethyl $-4,4^{\prime}-$ dimethoxy $-5,6,5^{\prime}, 6^{\prime}-$ dimethlene - dioxybiphenyl - 2,2 - dicarboxylate) is important drug of medicine not expensive since large number of people are using it in virus $\mathrm{B}$ and $\mathrm{C}$ cases for very large periods extend to many years.

The protective of DDB on chemically induced damage was studied in primary cultures of mammals hepatocytes.

Results:This work study of cytogenetic and biochemical effect of DDB, in mice using the chromosomes of bone marrow of male and pregnant female shown some changes with liver embryos. Also germ cells of testes given non significant aberration when compared with control.

As well as some biochemical parameters in serum and tissues, shown non significant changes in nucleic acid, total protein, total cholesterol, total glucose, total triglycerides and lactate dehydrogenase (LDH). Also, enzyme analysis of liver function and kidney.
\end{abstract}

\section{Introduction}

Investigation of DDB (Dimethly dimethoxy biphenyl Dicarboxylate) is synthetic analogue of schisandrin $\mathrm{C}$ which is a traditional Chinese medicine since 1977 and was tried in treatment of chronic HCV in china and Egypt with encouraging results (Montasser 2000 and 2001).

DDB with the chemical structure given below has been used for the treatment of viral hepatitis and drug - induced liver injury in china for about ten years.

Liver represents the largest organ in the mammaline body. DDB had been shown to be able to protect the liver against hepatotoxins such as $\mathrm{CCL}_{2}$, and thioacetamide to induce liver microsomal cytochrome P-450 in mice and rats ( Liu et al 1997, 1982 and Liu and Lesca 1982).

This drug was also shown to inhibit the mutagenic action of benzo pyrene (BP) and aflatoxine $\left(\mathrm{AFB}_{1}\right)$ in Ames test (Liu and Lesca 1982 \& Wang 1984) and to inhibit $\left(\mathrm{AFB}_{1}\right)$ - induced hepatocarcinogenesis in rats (Yan et al, 1986).

DDB which is a synthetic analogue to schisandrin $\mathrm{C}$ (active ingredient in schisandra chinesis extract) showed the powerful hepatoprotective and antiviral activity (Gao et al, 2005). It was frequently used in Egypt in the management of chronic viral and non-viral hepatitis. It showed reduction of hepatocellular carcinoma thought the decrease of alpha-fetoprotein levels (Montaser, 1999).

Treatment options for common liver disease such as cirrhosis, faty liver chronic hepatitis. In china DDB has been tested clinically scince Liu (1979) on patients with viral hepatitis B. The results indicate that DDB markedly improve impaired liver function. Similarly Mak and Ko (1997) Suggested that DDB had hepatoprotective effect on $\mathrm{CCL}_{4}$ - induced liver toxicity.

However Kim and Colleagues (1999) investigated the effect of DDB and observed that either single or repeated DDB pretreatment did not alter hepatotoxicity induced by $\mathrm{CCL}_{4}$.

This work aims to study the cytogenetic and biochemical effects of DDB (Bifendate pilutes) in mice using the chromosomes of bone marrow cells, liver embryoes and germ cells.

Some biochemical parameters like Nucleic acid protein and enzymes were analyzed. 


\section{Material and Methods}

\section{Chemicals:}

Definition of drug:Trade name : DDB

Generic name: Bifendate Pilules

Chemical name and structural formula: Chemical name: Dimethyl - 4,4' dimethoxy - 5,6,5', $6^{\prime}$ - dimethylenedioxy biphenyl - 2,2 dicarboxylate

Molecular formula: $\mathrm{C}_{20} \mathrm{H}_{18} \mathrm{O}_{10}$

Molecular weight: 418.36

Structural formula :<smiles>COC(=O)c1cc(OC)c2c(c1-c1c(C(=O)OC)cc(OC)c3c1OCO3)OCO2</smiles>

Category: Antihepatitis agent

Kits: Glutamic - pyruvic transaminase GPT (ALT)

Glutamic - oxaloacetic transaminase GOT (AST)

Kit, Total protein, Cholesterol, glucose, triglycerides

Kit, Creatinina and Urea Kit (C cromastes linear chemicals S.T.)

Statistical analysis were performed using un paired t-testes (Sokall and Rohlf 1969).

\section{Animals:}

80 adult fertile male and adult virgin female Swiss albino mice 8-12 weeks old weighted between 25-30 g were used from the Department of Animal House colony of National Research Center.

All animals used in this study is divided into two main part:

First part: Cytogenetic part

Second part: Biochemical analysis

Experiment were carried out to evaluate the effect of DDB drug using different cytogenetic study and Biochemical parameters.

Animals were divided into two main equal group:

Group I : 20 animals (10 male and 10 pregnant female mice) which considered untreated mice or normal standard were gives distilled water for 30 days.

Group II: 20 animals (10 male and 10 pregnant female mice-were fed orally by DDB at $0.75 \mathrm{mg} / \mathrm{kg} /$ day for 30 days.

After the last dose animals were scarified.

\section{A. Cytogenetic Part:}

Chromosomes preparation for 40 male and pregnant female mice were caged individually and were randomly divided into two groups:

Group I : 10 male and 10 pregnant female mice served as control and were administrated with $(0.25 \mathrm{ml})$ distilled water.

Group II: 10 male and 10 pregnant female mice were orally administrated with $(0.75 \mathrm{mg} / \mathrm{kg}$ / day) DDB for 30 day. (with 5 embryos for each mother).

In each mice (male and pregnant female mice) study somatic chromosomal aberration was made for bone marrow (Yosida et al, 1971) with liver lived embryos of mother treated DDB and control (50 embryo each group) according to (Romagnano et al., 1985).

In each male study germ cells (Spermatocytes) according to (Evans et al., 1964).

\section{B. Biochemical Part:}

For determined biochemical parameters for 40 male and pregnant female mice with their embryos. 40 animals were used in form of two groups as same as cytogenetic part.

The blood of mice (male and pregnant female mice) was collected and serum was separated to determine serum glucose (Trinder, 1969); Triglycerides (Fassati and Prencipe, 1982); Cholesterol (Richmond, 1973); Liver enzyme GPT (ALT) and GOT (AST) according to Reitman and Frankel (1975); Kidney enzyme Creatnine (Bartles et al, 1972) and Urea (Fawcett and Soctt., 1960).

Nucleic acid and total protein were determined in different tissues (Liver, Kidney, Testes and Liver embryos). DNA (Peares, 1985), RNA (Schneider 1957) and Total protein according to (Peter, 1968). 


\section{Results}

\section{I- Cytogenetic effect of DDB in mice:}

\section{Effect on males}

The cytogenetic effect of male shown in table $(1,2)$. The main types of structural chromosomal aberration are gaps, breaks, centromeric attenuations (C.A) and endomitosis in somatic cell of bone morrow. Table (1) showed that DDB (0.75 $\mathrm{mg} / \mathrm{kg} / \mathrm{b} . \mathrm{w})$ did not produce any significant change of chromosomal aberration than control in somatic cell. Table (2) shown the effect of DDB in the same male mice after 30 days in germ cells (spermatocytes) (x-y univalent, Autosmal univalent and chain). It is clear that DDB alone did not caused significant change when compared with control.

\section{Effect on pregnant females:}

In pregnant mice given DDB $(0.75$ $\mathrm{mg} / \mathrm{kg} / \mathrm{b} . \mathrm{w}$ ) daily for 30 days (Table 3 ) the result indicate non significant aberration on somatic cells (structural and numerical) when compared with untreated mice.

Table (4) shown the changes of structural chromosomal aberration in liver embryo of treated mother with DDB $(0.75$ $\mathrm{mg} / \mathrm{kg} / \mathrm{b} . \mathrm{w}$ ) when compared with control embryo there is non significant changes.

Mitotic index in all tables where a non significant difference appeared with treated mice (DDB) than control mice.

\section{II-Biochemical effect of DDB in mice:}

\section{Changes in DNA, RNA and protein.}

The results were absorved the effect of DDB on DNA, RNA and protein in different tissue of male mice (Table 5). There were some different but this diferents was non significant than control in all parameters.

As same as in pregnant female and liver embryos the effect of DDB on DNA, RNA and protein presented in table (6). The changes in all prameters gives non significant change when compared with control mother and embryo.

\section{Effect of DDB in Enzymatic serum mice:}

On the other hand, from male and pregnant female mice that exposure to DDB daily oral $(0.75 \mathrm{mg} / \mathrm{kg} / \mathrm{d} . \mathrm{w})$ for 30 days results of Enymatic serum (glucose, Triglycerides, cholesterol and LDH) (Table 7) did not give any changes than untreated mice which considered standard value.

\section{Effect of DDB in liver function of mice:}

In this result we studied the effect of DDB drug in tissues and serum of mice by determined the value liver enzymes GOT (AST) and GPT (ALT) in (Table 8, 9).

The result indicate there were decrease in the value of tissues (liver male, liver pregnant female an liver embryo) and serum (male and pregnant female) than control mice.

\section{Effect of DDB in Kidney function of mice:}

Table (10) indicate the serum of male and pregnant female mice which treated with DDB give nonsignificant data in creatinine and urea when compared with control. 
Table (1): Effect of DDB on chromosomes of bone marrow (somatic cells) in male mice after 30 days)

\begin{tabular}{|c|c|c|c|c|c|c|c|c|c|c|c|c|c|}
\hline \multirow{3}{*}{$\begin{array}{c}\text { Treatm } \\
\text { ent }\end{array}$} & \multirow{3}{*}{$\begin{array}{l}\text { No of } \\
\text { mice }\end{array}$} & \multirow{3}{*}{$\begin{array}{l}\text { No of } \\
\text { Cells } \\
\text { scord }\end{array}$} & \multicolumn{6}{|c|}{ Structural Aberration } & \multicolumn{2}{|c|}{$\begin{array}{l}\text { Numerical } \\
\text { aberration }\end{array}$} & \multicolumn{3}{|c|}{ Mitotic Index } \\
\hline & & & \multirow{2}{*}{ Break } & \multirow{2}{*}{$\begin{array}{l}\text { End } \\
\text { mitosis }\end{array}$} & \multirow{2}{*}{$\begin{array}{l}\text { C. } \\
\text { A }\end{array}$} & \multirow{2}{*}{$\begin{array}{c}\text { Ga } \\
\mathbf{p}\end{array}$} & \multicolumn{2}{|c|}{$\begin{array}{c}\text { Total } \\
\text { excluding gap }\end{array}$} & \multirow{2}{*}{$\begin{array}{l}\text { Hy } \\
\text { po }\end{array}$} & \multirow{2}{*}{ Hyper } & \multirow{2}{*}{$\begin{array}{l}\text { No of } \\
\text { cells }\end{array}$} & \multirow{2}{*}{$\%$} & \multirow{2}{*}{$\underset{\text { S.E. }}{\text { Mean } \pm}$} \\
\hline & & & & & & & $\%$ & $\begin{array}{l}\text { Mean } \pm \\
\text { S.E. }\end{array}$ & & & & & \\
\hline Control & 10 & 500 & $0.4 \%$ & $0.4 \%$ & $\begin{array}{l}1 \\
\%\end{array}$ & $\begin{array}{l}0.6 \\
\%\end{array}$ & $\begin{array}{l}1.8 \\
\%\end{array}$ & $\begin{array}{c}3 \pm \\
0.866\end{array}$ & -- & -- & 10000 & $\begin{array}{c}34.7 \\
\%\end{array}$ & $\begin{array}{c}347 \pm \\
15.004\end{array}$ \\
\hline $\begin{array}{l}\text { DDB } \\
\text { dray }\end{array}$ & 10 & 500 & $0.4 \%$ & $0.4 \%$ & 1 & $\begin{array}{l}0.8 \\
\%\end{array}$ & $\begin{array}{l}2.2 \\
\%\end{array}$ & $\begin{array}{c}3.67 \pm \\
0.577\end{array}$ & $\begin{array}{l}0.2 \\
\%\end{array}$ & $0.2 \%$ & 10000 & $\begin{array}{c}33.8 \\
\%\end{array}$ & $\begin{array}{r}338 \pm \\
18.843\end{array}$ \\
\hline
\end{tabular}

Table (2): Effect of DDB on chromosomes of testes (Spermatocytes) in male mice after 30 days.

\begin{tabular}{|l|c|c|c|c|c|c|c|}
\hline \multirow{2}{*}{ Treatment } & \multirow{2}{*}{$\begin{array}{c}\text { No of examined } \\
\text { male mice }\end{array}$} & \multirow{2}{*}{$\begin{array}{c}\text { No of cells } \\
\text { scord }\end{array}$} & $\begin{array}{c}\text { X-Y } \\
\text { univalent }\end{array}$ & $\begin{array}{c}\text { Autosomal } \\
\text { univalent }\end{array}$ & Chain & \multirow{2}{*}{$\begin{array}{c}\text { Total } \\
\text { Mean } \pm \text { S.E. }\end{array}$} & \\
\hline Control & 10 & 500 & $\mathbf{0 . 6 \%}$ & $\mathbf{0 . 8 \%}$ & -- & $\mathbf{1 . 4 \%}$ & $\mathbf{2 . 3 3} \pm \mathbf{1 . 4 7 2}$ \\
\hline DDB dray & $\mathbf{1 0}$ & $\mathbf{5 0 0}$ & $\mathbf{0 . 8 \%}$ & $\mathbf{0 . 8 \%}$ & $\mathbf{0 . 2 \%}$ & $\mathbf{1 . 8 \%}$ & $\mathbf{3} \pm \mathbf{1 . 2 2 5}$ \\
\hline
\end{tabular}

Table (3): Effect of DDB on chromosomes of bone marrow in pregnant female mice after 30 days.

\begin{tabular}{|c|c|c|c|c|c|c|c|c|c|c|c|c|c|}
\hline \multirow{3}{*}{$\begin{array}{c}\text { Treatm } \\
\text { ent }\end{array}$} & \multirow{3}{*}{$\begin{array}{c}\text { No } \\
\text { of } \\
\text { mice }\end{array}$} & \multirow{3}{*}{$\begin{array}{c}\text { No } \\
\text { of } \\
\text { Cell } \\
\text { s } \\
\text { scor } \\
\text { d }\end{array}$} & \multicolumn{6}{|c|}{ Structural Aberration } & \multicolumn{2}{|c|}{$\begin{array}{l}\text { Numerical } \\
\text { aberration }\end{array}$} & \multicolumn{3}{|c|}{ Mitotic Index } \\
\hline & & & \multirow{2}{*}{$\begin{array}{l}\text { Bre } \\
\text { ak }\end{array}$} & \multirow{2}{*}{$\begin{array}{c}\text { End } \\
\text { mito } \\
\text { sis }\end{array}$} & \multirow{2}{*}{ C.A } & \multirow{2}{*}{ Gap } & \multicolumn{2}{|c|}{$\begin{array}{l}\text { Total excluding } \\
\text { gap }\end{array}$} & \multirow{2}{*}{$\begin{array}{c}\text { Hyp } \\
\text { о }\end{array}$} & \multirow{2}{*}{ Hyper } & \multirow{2}{*}{$\begin{array}{l}\text { No } \\
\text { of } \\
\text { cells }\end{array}$} & \multirow{2}{*}{$\%$} & \multirow{2}{*}{$\begin{array}{l}\text { Mean } \pm \\
\text { S.E. }\end{array}$} \\
\hline & & & & & & & $\%$ & $\begin{array}{l}\text { Mean } \pm \\
\text { S.E. }\end{array}$ & & & & & \\
\hline $\begin{array}{l}\text { Contro } \\
\text { l }\end{array}$ & 10 & 500 & $\begin{array}{c}0.6 \\
\%\end{array}$ & $\begin{array}{l}1.2 \\
\%\end{array}$ & $\begin{array}{l}1.6 \\
\%\end{array}$ & $0.6 \%$ & $3.4 \%$ & $6 \pm 1.414$ & -- & -- & $\begin{array}{c}1000 \\
0\end{array}$ & $\begin{array}{c}27.85 \\
\%\end{array}$ & $\begin{array}{c}248.5 \pm \\
6.139\end{array}$ \\
\hline $\begin{array}{l}\text { DDB } \\
\text { dray }\end{array}$ & 10 & 500 & $1 \%$ & $\begin{array}{l}1.4 \\
\%\end{array}$ & $\begin{array}{l}1.8 \\
\%\end{array}$ & $0.8 \%$ & $4.2 \%$ & $7 \pm 1.414$ & $\begin{array}{c}0.2 \\
\%\end{array}$ & $0.2 \%$ & $\begin{array}{c}1000 \\
0\end{array}$ & $\begin{array}{c}27.65 \\
\%\end{array}$ & $\begin{array}{c}276.5 \pm \\
5.558\end{array}$ \\
\hline
\end{tabular}

Table (4): Effect of DDB on chromosomes mice liver embryo of treated mother after 30 days.

\begin{tabular}{|c|c|c|c|c|c|c|c|c|c|c|c|c|c|}
\hline \multirow{3}{*}{$\begin{array}{c}\text { Treatm } \\
\text { ent }\end{array}$} & \multirow{3}{*}{$\begin{array}{l}\text { No of } \\
\text { mice }\end{array}$} & \multirow{3}{*}{$\begin{array}{l}\text { No of } \\
\text { Cells } \\
\text { scord }\end{array}$} & \multicolumn{6}{|c|}{ Structural Aberration } & \multicolumn{2}{|c|}{$\begin{array}{l}\text { Numerical } \\
\text { aberration }\end{array}$} & \multicolumn{3}{|c|}{ Mitotic Index } \\
\hline & & & \multirow{2}{*}{ Breal } & \multirow{2}{*}{$\begin{array}{l}\text { End } \\
\text { mitosis }\end{array}$} & \multirow{2}{*}{ C.A } & \multirow{2}{*}{ Gap } & \multicolumn{2}{|c|}{$\begin{array}{c}\text { Total excluding } \\
\text { gap } \\
\end{array}$} & \multirow{2}{*}{ Hypo } & \multirow{2}{*}{ Hyper } & \multirow{2}{*}{$\begin{array}{l}\text { No of } \\
\text { cells }\end{array}$} & \multirow{2}{*}{$\%$} & \multirow{2}{*}{$\begin{array}{l}\text { Mean } \\
\pm \text { S.E. }\end{array}$} \\
\hline & & & & & & & $\%$ & $\begin{array}{l}\text { Mean } \pm \\
\text { S.E. }\end{array}$ & & & & & \\
\hline Control & 50 & 2500 & $\begin{array}{c}0.36 \\
\%\end{array}$ & $0.2 \%$ & $\begin{array}{l}0.6 \\
4 \%\end{array}$ & $\begin{array}{c}0.28 \\
\%\end{array}$ & $\begin{array}{l}1.2 \\
\%\end{array}$ & $10 \pm 3.938$ & -- & -- & 50000 & $\begin{array}{c}8.3 \\
\%\end{array}$ & $\begin{array}{c}415 \pm \\
12.247\end{array}$ \\
\hline $\begin{array}{l}\text { DDB } \\
\text { dray }\end{array}$ & 50 & \begin{tabular}{|l|}
2500 \\
\end{tabular} & $\begin{array}{c}0.52 \\
\%\end{array}$ & $0.24 \%$ & $\begin{array}{l}0.8 \\
4 \% \\
\end{array}$ & $\begin{array}{c}0.32 \\
\%\end{array}$ & $\begin{array}{l}1.6 \\
\% \\
\end{array}$ & $\begin{array}{l}13.33 \pm \\
5.308 \\
\end{array}$ & $\begin{array}{c}0.28 \\
\%\end{array}$ & $0.32 \%$ & 50000 & $\begin{array}{c}8.16 \\
\%\end{array}$ & $\begin{aligned} 408 \pm \\
12.151\end{aligned}$ \\
\hline
\end{tabular}


Table (5): Effect of DDB on DNA, RNA and protein in male mice after 30 days.

\begin{tabular}{|l|c|c|c|c|c|c|}
\hline \multirow{2}{*}{ Treatment } & \multicolumn{3}{|c|}{ Control } & \multicolumn{3}{c|}{ DDB } \\
\cline { 2 - 7 } & Liver & Kidney & Testes & Liver & Kidney & Testes \\
\hline Total & $\mathbf{0 . 4 1 8} \pm$ & $\mathbf{0 . 3 0 3} \pm$ & $\mathbf{0 . 3 1 2} \pm$ & $\mathbf{0 . 4 2 6} \pm$ & $\mathbf{0 . 3 0 9} \pm$ & $\mathbf{0 . 3 2 3} \pm$ \\
DNA mg/g & $\mathbf{0 . 0 0 5}$ & $\mathbf{0 . 0 6 4}$ & $\mathbf{0 . 0 2 3}$ & $\mathbf{0 . 0 0 5}$ & $\mathbf{0 . 0 1}$ & $\mathbf{0 . 0 0 5}$ \\
\hline Total & $\mathbf{0 . 2 7 1} \pm$ & $\mathbf{0 . 1 7 8} \pm$ & $\mathbf{0 . 1 9 0} \pm$ & $\mathbf{0 . 2 7 6} \pm$ & $\mathbf{0 . 1 8 6} \pm$ & $\mathbf{0 . 1 9 3} \pm$ \\
RNA $\mathbf{~ m g / g ~}$ & $\mathbf{0 . 0 3 3}$ & $\mathbf{0 . 0 0 6}$ & $\mathbf{0 . 0 2 1}$ & $\mathbf{0 . 0 3 5}$ & $\mathbf{0 . 0 2 1}$ & $\mathbf{0 . 0 0 5}$ \\
\hline Total & $\mathbf{7 . 0 4 1} \pm$ & $\mathbf{4 . 9 5 5} \pm$ & $\mathbf{4 . 4 3 6} \pm$ & $\mathbf{7 . 1 2 6} \pm$ & $\mathbf{4 . 9 5 9} \pm$ & $\mathbf{4 . 5 2 7} \pm$ \\
Protein $\mathbf{g} / \mathbf{g}$ & $\mathbf{0 . 1 6 1}$ & $\mathbf{0 . 1 8 3}$ & $\mathbf{0 . 1 3 7}$ & $\mathbf{0 . 2 0 8}$ & $\mathbf{0 . 1 1 8}$ & $\mathbf{0 . 1 4 3}$ \\
\hline
\end{tabular}

Table (6): Effect of DDB on DNA, RNA and protein in pregnant female and their embryos after 30 days.

\begin{tabular}{|l|c|c|c|c|c|c|}
\hline \multirow{3}{*}{ Treatment } & \multicolumn{3}{|c|}{ Control } & \multicolumn{3}{c|}{ DDB } \\
\cline { 2 - 7 } & Liver & Kidney & Liver & Liver & Kidney & Liver \\
& & & embryo & & \\
\hline Total & $\mathbf{0 . 3 6 9} \pm$ & $\mathbf{0 . 2 5 2} \pm$ & $\mathbf{0 . 2 0 8} \pm$ & $\mathbf{0 . 3 7 8} \pm$ & $\mathbf{0 . 2 8 2} \pm$ & $\mathbf{0 . 2 1 9} \pm$ \\
DNA mg/g & $\mathbf{0 . 0 0 6}$ & $\mathbf{0 . 0 0 9}$ & $\mathbf{0 . 0 0 4}$ & $\mathbf{0 . 0 0 5}$ & $\mathbf{0 . 0 0 6}$ & $\mathbf{0 . 0 0 4}$ \\
\hline Total & $\mathbf{0 . 2 3 9} \pm$ & $\mathbf{0 . 1 5 6} \pm$ & $\mathbf{0 . 1 3 7} \pm$ & $\mathbf{0 . 2 4 5} \pm$ & $\mathbf{0 . 1 5 8} \pm$ & $\mathbf{0 . 1 4 7} \pm$ \\
RNA mg/g & $\mathbf{0 . 0 1 2}$ & $\mathbf{0 . 0 1 1}$ & $\mathbf{0 . 0 0 8}$ & $\mathbf{0 . 0 1 4}$ & $\mathbf{0 . 0 0 8}$ & $\mathbf{0 . 0 1 4}$ \\
\hline Total & $\mathbf{7 . 0 2 7} \pm$ & $\mathbf{4 . 5 7 9} \pm$ & $\mathbf{3 . 0 5 2} \pm$ & $\mathbf{7 . 1 1 1} \pm$ & $\mathbf{4 . 5 8 1} \pm$ & $\mathbf{3 . 1 1 2} \pm$ \\
Protein $\mathbf{g} / \mathbf{g}$ & $\mathbf{0 . 1 8 8}$ & $\mathbf{0 . 1 6 6}$ & $\mathbf{0 . 1 9 6}$ & $\mathbf{0 . 1 3 4}$ & $\mathbf{0 . 1 6 1}$ & $\mathbf{0 . 1 7 4}$ \\
\hline
\end{tabular}

Table (7): Effect of DDB on biochemical markers in male and pregnant female mice after 30 days.

\begin{tabular}{|l|c|c|c|c|}
\hline \multirow{2}{*}{ Treatment } & \multicolumn{2}{c|}{ Male } & \multicolumn{2}{c|}{ Pregnant Female } \\
\cline { 2 - 5 } & Control & DDB & Control & DDB \\
\hline Glucose $\mathrm{mg} / \mathrm{dl}$ & $\mathbf{8 9 . 1 2 1} \pm \mathbf{0 . 5 8 6}$ & $\mathbf{8 9 . 1 1 8} \pm \mathbf{0 . 6 2 9}$ & $\mathbf{7 9 . 8 3} \pm \mathbf{0 . 5 8 3}$ & $\mathbf{7 9 . 6 6 2} \pm \mathbf{0 . 5 8 7}$ \\
\hline Triglycerides mg/dl & $\mathbf{6 6 . 3 3 9} \pm \mathbf{1 . 4 1 2}$ & $\mathbf{6 6 . 0 2 4} \pm \mathbf{1 . 5 3 4}$ & $\mathbf{5 0 . 8} \pm 4.269$ & $\mathbf{4 9 . 6} \pm \mathbf{2 . 7 8 2}$ \\
\hline Cholesterol $\mathrm{mg} / \mathrm{dl}$ & $193.836 \pm 1.556$ & $193.64 \pm 1.583$ & $\mathbf{1 8 4 . 3 2 8} \pm \mathbf{1 . 5 6 4}$ & $\mathbf{1 8 4 . 1 9 2} \pm \mathbf{1 . 6 3 9}$ \\
\hline LDH U/L & $\mathbf{2 5 9 . 6 9 2} \pm \mathbf{5 . 0 0 4}$ & $\mathbf{2 5 8 . 7 2 3} \pm \mathbf{5 . 7 0 7}$ & $\mathbf{2 5 0 . 0 3 3} \pm \mathbf{5 . 6 5 1}$ & $\mathbf{2 4 7 . 0 9 5} \pm \mathbf{5 . 1 0 7}$ \\
\hline
\end{tabular}

Table (8): Effect of DDB on liver enzymes in tissues male and pregnant female with their embryos after 30 days.

\begin{tabular}{|l|c|c|c|c|c|c|}
\hline \multirow{3}{*}{ Treatment } & \multicolumn{4}{|c|}{ Control } & \multicolumn{3}{c|}{ DDB } \\
\cline { 2 - 7 } & Liver male & $\begin{array}{c}\text { Liver pregnant } \\
\text { female }\end{array}$ & Liver embryo & Liver male & $\begin{array}{c}\text { Liver pregnant } \\
\text { female }\end{array}$ & $\begin{array}{c}\text { Liver } \\
\text { embryo }\end{array}$ \\
\hline GOT(AST) & $\mathbf{5 2 . 4} \pm$ & $\mathbf{4 5 . 6} \pm$ & $\mathbf{3 9 . 5} \pm$ & $\mathbf{5 0 . 4} \pm$ & $\mathbf{4 3 . 7} \pm$ & $\mathbf{3 9} \pm$ \\
U/L & $\mathbf{2 . 2 3 4}$ & $\mathbf{1 . 4 5 9}$ & $\mathbf{1 . 6 8 7}$ & $\mathbf{3 . 1 5 9}$ & $\mathbf{1 . 8 5 3}$ & $\mathbf{1 . 9 5 6}$ \\
\hline GPT (ALT) & $\mathbf{5 8 . 8} \pm$ & $\mathbf{5 6 . 1} \pm$ & $\mathbf{4 3 . 8} \pm$ & $\mathbf{2 5 . 5 3} \pm$ & $\mathbf{5 2 . 5 3} \pm$ & $\mathbf{4 3} \pm$ \\
U/L & $\mathbf{1 . 9 6}$ & $\mathbf{2 . 2 4 1}$ & $\mathbf{0 . 9 9 1}$ & $\mathbf{1 . 2 5 1}$ & $\mathbf{1 . 3 4 3}$ & $\mathbf{0 . 8 6 1}$ \\
\hline
\end{tabular}

Table (9): Effect of DDB on liver enzymes male and pregnant female in serum after 30 days.

\begin{tabular}{|c|c|c|c|c|}
\hline \multirow{2}{*}{ Treatment } & \multicolumn{2}{|c|}{ Control } & \multicolumn{2}{c|}{ DDB } \\
\cline { 2 - 5 } & Male & Pregnant female & Male & Pregnant female \\
\hline GOT(AST) U/L & $\mathbf{6 5} \pm \mathbf{2 . 2 2 2}$ & $\mathbf{6 3 . 4} \pm \mathbf{2 . 9 2 4}$ & $\mathbf{6 2 .} \pm \mathbf{1 . 7 5 7}$ & $\mathbf{6 1 . 3} \pm \mathbf{1 . 1 1 2}$ \\
\hline GPT(ALT) U/L & $\mathbf{6 7 . 5 5} \pm \mathbf{1 . 5 6 5}$ & $\mathbf{6 6 . 2} \pm \mathbf{2 . 2 3 2}$ & $\mathbf{6 4 . 9} \pm \mathbf{2 . 6 1 6}$ & $\mathbf{6 3} \pm \mathbf{2 . 8 4 1}$ \\
\hline
\end{tabular}


Table (10): Effect of DDB on kidney function male and pregnant female in serum after 30 days.

\begin{tabular}{|l|c|c|c|c|}
\hline \multirow{2}{*}{ Treatment } & \multicolumn{2}{|c|}{ Creatinine } & \multicolumn{2}{c|}{ Urea } \\
\cline { 2 - 5 } & Male & Pregnant female & Male & Pregnant female \\
\hline Control & $\mathbf{0 . 8 9 6} \pm \mathbf{0 . 0 1 6}$ & $\mathbf{0 . 7 8 8} \pm \mathbf{0 . 0 1 8}$ & $\mathbf{1 9 . 2 7 9} \pm \mathbf{0 . 3 7 8}$ & $\mathbf{1 6 . 7 9 6} \pm \mathbf{0 . 4 2 8}$ \\
\hline DDB & $\mathbf{0 . 8 4 3} \pm \mathbf{0 . 0 2 3}$ & $\mathbf{0 . 7 3 3} \pm \mathbf{0 . 0 2 1}$ & $\mathbf{1 8 . 3 4 4} \pm \mathbf{0 . 3 8}$ & $\mathbf{1 5 . 3 0 1} \pm \mathbf{0 . 4 4 3}$ \\
\hline
\end{tabular}

\section{Discussion}

The present study showed the effect of DDB drug on mice (male, Pregnant female and their embryos). In order to investigated the role of DDB in mammals because $\mathrm{HCV}$ infection is wide spread problem following mainly blood transfusion, surgical procedures, operations and dental procedure (Donaldson et al, 1994).

Lenord (2005) shown that the DDB is a beneficial effect on mammal. The changes of chromosomal mice (male, Pregnant female and their embryos) non significant which mean safty and no mutagenic action was detected.

IP et al (2000) added that the treating mice with DDB daily oral dose did not produce any significant alteration in plasma alanine amino transferase (ALT) and sorbital dehydrogenase (SDH) activity. Also, Lui et al (2005) and Goa et al (2005) supported that the reduced elevated on ALT and AST on serum and Tissues after treated with DDB drug. Adding to Lui (1989) which said that the DDB improved the liver function in female rats as the elevated serum, GPT and GOT in liver hepatities patients have been decreased.

In carcinogen-induce DNA damage that the DDB is able to directly or indirectly protective effect (Ging and Liue, 1992) and Gao et al 2005). Also, Chang et al (2004) and Gao et al (2005) added that the DDB protect the inhibition of RNA which agreement with our results.

$\mathrm{Fu}$ and Liu (1992) reported that when normal rats were given DDB daily for 10 days, the free ribosomal protein and RNA liver increased significantly. Also, liver glycogen and blood glucose was reduced with DDB.

Mowafy (2004) investigated that the DDB when given befor meal to patients with chronic hepatitis $\mathrm{C}$ which is big problem in our country. The ALT and AST is lowing in serum and no effects on blood urea and serum creatinine.

The protective action of the drug mainly referred to its corrective action on protein synthesis with repair of the structure and function of damaged hepatocytes (Xa et al 1997).

This study concluded that the DDB effective observed no side effect (Salame et al 2004) which equal to our results. Also, DDB caused a significant reduction in elevated levels of all serum enzymes (ALT, AST, LDH and SDH) compared with levels of $\mathrm{CCL}_{4}$.

This improvement of impair liver function suggests that the DDB could be used for treatment of chronic viral hepatitis $\mathrm{B}$ in human as it has been to reduce the main symptoms of patients and its side effect are rare and not serious (El Saway et al 2002).

In conclusion, the present data indicate that oral administration of DDB, have a beneficial effects on damaged liver cells to prevent lipid peroxidation and improve antioxidant enzyme activiles.

The data obtained from study revealed that DDB commediate its biochemical effects to protect action against liver.

The toxicity of DDB is very rare No teratogenic or mutagenic action was detected No untoward effects of DDB have been observed. DDB is not expensive with no side effects.

\section{References}

1. Bartles $\mathbf{H}$, Bohmer $M$ and Herirli $C$ (1972): Creatinine coloimetric kinetic Method Clin - Chem. Acta, 37, 193 - 195.

2. Chang SM, Kuhn J, Wen P, Greenberg H, Schiff D, Conrad C, Fink K, Robins HI, Cloulghesy T, De Angelis L, Rozier J, Hess K, Dancey $J$ and Prados MD 
(2004): Phase 1 / Pharmacokienetic Study of CCL-779 in Patients with recurrent malignant glioma on enzyme - inducing antiepileptic durgs 1-Investigational New drug, 22 (4):427-432.

3. Donaldson P, Doherty $D$ and LInder hill J (1994): The molecular genetic of autoimmune liver disease. Hepatology, 20:225 - 230 .

4. El- Beshbishy HA (2005): The effect of Dimethyl Dimethoxy Biphenyl Dicarobxylate (DDB) against Tamoxifen induced liver injury in Rats: DDB use Is curative or protective. J. of Biochemistry and Molecular Biology, 38 (3): 300 - 306.

5. El-Saway SA, El-Shafey AM and ElBahrawy HA (2002): Effect of dimethly diphenyl bicarboxylate on normal and chemically injured liver. Estern Mediterranean health Journal, 8 (1).

6. Evans E P, Breckmon $\mathbf{G}$ and Ford $\mathbf{C F}$ (1964): An-air drying method for meiotic preparation from mammalian tests. Cytogentics, 3: 289 - 291.

7. Fassati P and Prencipe L. (1982): Trigly cerides Enzymetic colorimetric method. Clin. Chem., 28: 2077-2081.

8. Fawcette JK and Soctt JT (1966): Urease - Berthelot Method J. clin path, 13: 156 159.

9. Fu T and Liu G (1994) : Protective effects of dimethy $\mathrm{L}-4.4^{\prime}-$ dimethoxy $-5,6,5^{\prime}, 6^{\prime}$ - dimethylene dioxybiphenyl - 2, $2^{\prime}$ dicarloxylene on damages of isolated rat hepatocyte induced by carbon tetrachloride and D- galactosamine. Biomedical and environmental Science, 5 (3) : 185 - 194.

10. Gao M Zhang J and liu G (2005) : Effect of dipheuyl dimethyl bicarloxylate on cancanavalin A- induced liver injury in mice. Lin - Int , 25 (4) : $904-912$.

11. IP SP, yiu $H \quad Y$ and KO KH (2000): Differential effect of schisandrin $\mathrm{B}$ and dimethyl diphenyl bicarloxylate (DDB) on hepatic mitochondrial glutathione redo status in carbon tetrachloride intoxicated mice. Mol cell Biochem 205 (1-2): 111-114

12. Kim S N, Kim sy, yim HK, lee w y Han $\mathrm{K}-\mathrm{S}$,Kim SK, yoon $\mathbf{N} \mathbf{y}$ and Kim y c (1999): Effect of dimethyl $-4,4^{\prime}-$ dimethoxy - 5,6,5', dimethylene dioxybiphenyl $-2,2^{\prime}$ dicarboxylare (DDB) on chemical - induced liver injry. Biol pharm Bul I , 22 (1) : 93 - 95.

13. Leonard A (2002): Hertable chromosome aberrations in mammals after exposure to chemicals. J. Radiation and Environmental Biophyscis, 13 (1) : $1-8$.

14. Liu G T, wamg GF, wei H L, Bao TT and song $Z Y$ (1979): Acomporison of the protective actions of Biphenyl Dime thyl Dicarloxylate, Trans - stillene, Alcoholic extracts of Fructus schizandra and ganoderma against experimental liver injury in mice. Acta pharm . sin, 14: $598-$ 604.

15. Liu $K$ T and Lesca $P$ (1982): Pharmacological properties of dibenzo (a) cyclooctine derivatives isolated from Fructus schizandra chinesis III. Inhibitory effects on carbon tetrachloride - induced lipid peroxidation metabolism and covalent binding of carbon tetrachloride to lipid. Chem. . Biol . Interact, 41: $39-47$.

16. Liu KT, Cresteil T, columelli $S$ and Lesca $P$ (1982): Pharmacological properties of dibenzo (a.c) cyclooctenc derivatives isolated from fructus schizandra chinesis II. Inductoon of phenobarbital. Like hepatic mono oxygenase Chem. Biol. Interact, 39: $312-330$.

17. Liu GT, Li y wei H L, Zhang H, Xu J y and yu LH (2005): Mechanism of protective action of bicyclol against CCL induced liver injury in mice. Liver internationall, 25, (4) : $872-879$.

18. Mak DH and Ko KM (1997). Alteration in susceptibility to carbon tetrachloride and hepatic antioxidant detoxification system in streptozotocin - induced short - term diabetic rats : Effect of insulin and schisandrin B treatment - Moleeular and cellular biochemistry, $17: 20$ (1-2) 225 - 232.

19. Mawafy T (2004). Clinical evaluation of efficacy of DDB on patients with chronic hepatitis $\mathrm{C} \mathrm{Al}$ Ahrame Company for drugs. Mol Cell Biochem 205 (4-2): 111-114.

20. Montaser M F (2000): Aclinical and Laboratory study of DDB in treatment of chronic hepatitis $\mathrm{C}: 2$ comparative studies. Ain shams Medical, J, 51 (1,2\&3) : 209 223.

21. Montaser M F (1999): Aclinical and labortory role of $\mathrm{DDB}$ in treatment of chronic hepatitis $\mathrm{C}$, a preliminary report in Egypt. Ain shams Med. J, 50 (12 - 12) : $1015-1023$.

22. Montasser $\mathbf{m}$ M F (2001): Clinical and laloratary studies of DDB in treatment of chronic Hepatitis Apilot studyin Egypt. Combination therapy with Amantadine Hydrochloride, Ain shams medical J. 52 $(1,2 \& 3): 125-142$.

23. Peares AGF (1985): Histochemistry theoretical and Applied, - volume two : Analytical technology, churchil livingston $4^{\text {th }}$ ed. Edinburgy London, Melbourne and New York.

24. Peter T (1968): proposals for standardization of total protein colorimetric 
method assays. Clin. Chem., 14 (12) : 1149 $-1159$.

25. Qing w and Liu G (1992): Protective effect of dimethyl - 4,4' - dimethoxy $5,6,5^{\prime}, 6^{\prime}$ - protective effect of dimethyl $2,3^{\prime}$ - dicarboxylate (DDB) against carcinogen induced ratinuclear DNA damage. Biomed Environ Sci, 5 (3): 201 - 207.

26. Reitman A and Frankel S (1957): Glutamic - pyruvic trans aminase (GPT) and Glutamic oxaloacetic transaminase (GOT). Am . J Clin-Path 28 : 56 - 63.

27. Richmond W (1973): Cholesterol enzymatic colorimetric method. Clin . Chem . $19: 1350-1355$.

28. Roeschlau P, Bernt $\mathbf{E}$ and Gruber w (1974): Enzymatic Determination of total cholesterol in serum: Z klin chem. Klin Biochem 12 (5) : $226-231$.

29. Romagnano A, King A w, Richer CL and Perrone M A (1985): Adirect technique for the preparation of chromosomes for early enquine embryos. Can, J. genet. Cytol $27: 365$ - 369.

30. Salama HM, Amer AR, Hammed OM, and El-Sayed WF (2004): Effect of DDB Monotherapy and in Combination with amantadine Hydrochloride and Ribavirin in patients with chronic Hepatitis $\mathrm{C}$ virus infection. Sci. Med. J. escme 16, 1:9 -16.

31. Schneider WC (1957): "Determination of nucleic acids in tissues by pentose analysis : In "Method enzymology" (Edited by colowick S.P. and Kaplan. N.O.) Academic Press. New York, 680-684 .

32. Sokall RR and Rohlf F J (1969): Biometry by W.H. ferman and Company Chapter, 9: 204-252.

33. Trinder P (1969): Glucose Enzymatic colorimetric method. Ann. Clin. Biochem, 6, 24-28.

34. VanuKumar MR, and Lathal MS (2002): Hepatoprotective effect of the methanolic extract of Curculigo orchioides in $\mathrm{CCL}_{4}$ treated male rats. Int. J. Pharmacol, 34: 269 $-275$.

35. Wang CF (1984): Mutagenetic and antimutagenetic effect of DDB Report on National Meeting for pharmacological society 1984.

36. Yan RQ, Chen ZY and Gin GZ (1986): Effect of Bifendati and three Chinese traditional medicinal herbs on the $\mathrm{AFB}_{1}$ induced hepatocarcinogenesis in rats. $\mathrm{J}$. Oncol, 5: 141-144.

37. Yosida TH, Truchiya $\mathbf{K}$ and Mariwaki K (1971): Frequency of chromosome polymorphism of Roltus Rattus collected in Japan. Chromosoma, 33: 30 - 40.

38. Zhanq BH, Gonq DZ and Mei MH (1999): Protection of regenerating liver after partial hepatectomy from carbon tetrachloride hepatotoxicity in rats: role of hepatic simulator substance. J. Gastrocuteral Hepatol, 14 (10): 1010 - 1017. 


\section{دراسة التأثير الوراثى الخلوى و الكيمياء الحيوى لعقار ال د د بى (Dیى (DDB)}

\section{على الجرزان البيضاء الصغيرة و أجنتها}

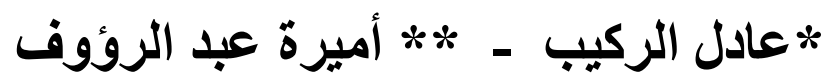

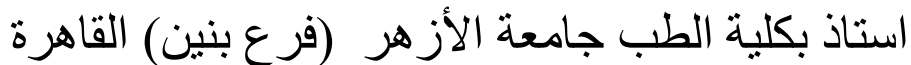

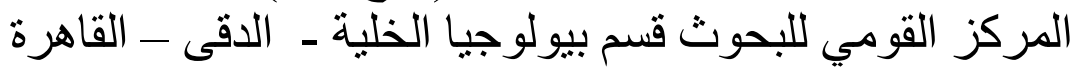

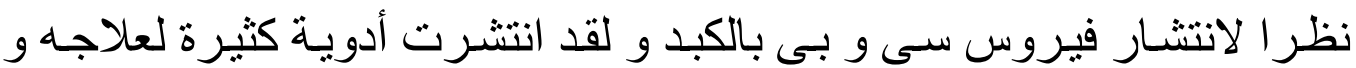

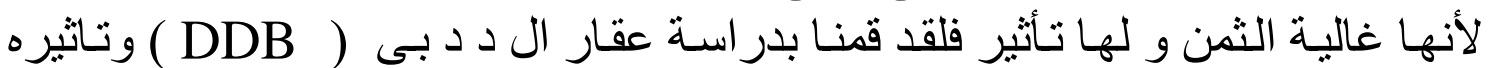

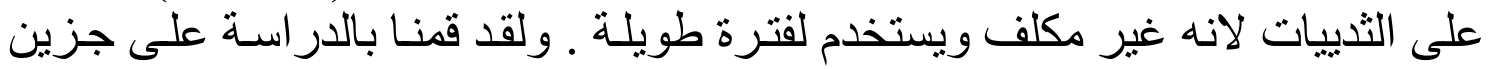

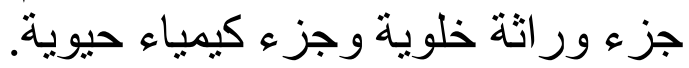

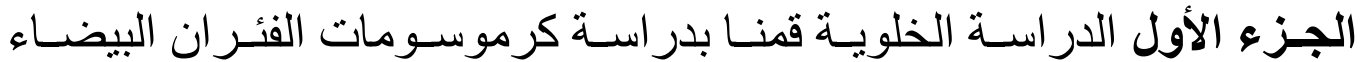

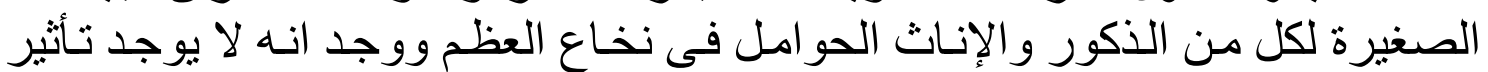

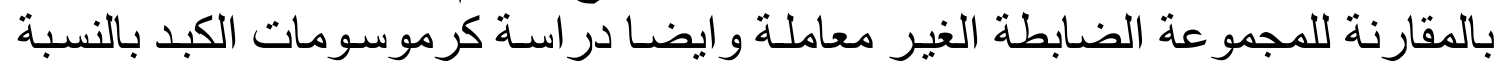

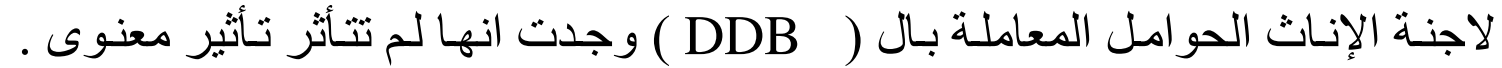

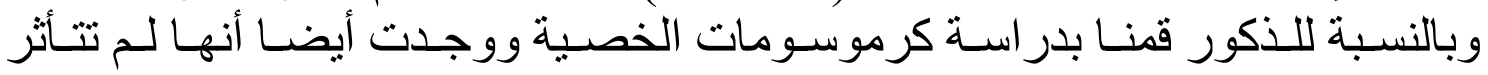
بالمقارنة بالمجموعة الغير معاملة .

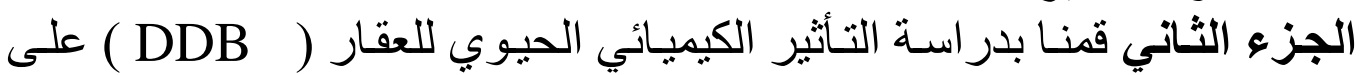

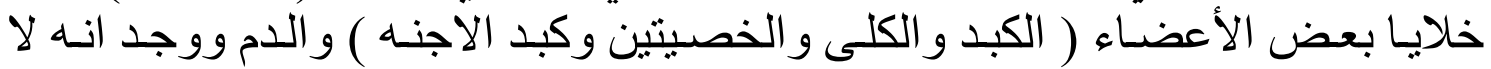

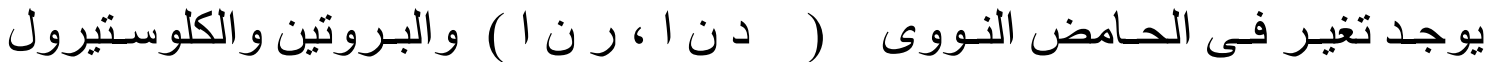

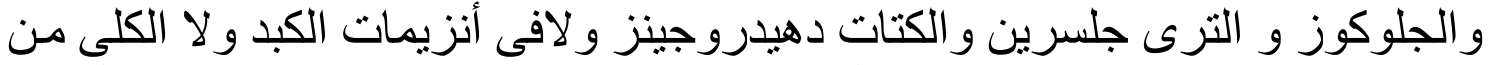

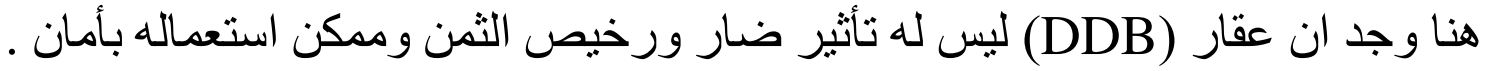

